\title{
Rigorous Density Functional Theory for Inhomogeneous Bose-Condensed Fluids
}

\author{
A. Griffin \\ Dipartimento di Fisica, Università di Trento, I-38050 Povo, Italy \\ and \\ Department of Physics, University of Toronto, Toronto, Ontario, Canada M5S 1A7\%
}

\begin{abstract}
To appear in the special Brockhouse Issue
of the Can. Journ. Phys., 1995.
\end{abstract}

Phone: 416-978-5199/7135

Fax: 416-978-2537

e-mail: griffin@physics.utoronto.ca

*permanent address 


\begin{abstract}
The density functional theory originally developed by Hohenberg, Kohn and Sham provides a rigorous conceptual framework for dealing with inhomogeneous interacting Fermi systems. We extend this approach to deal with inhomogeneous interacting Bose-condensed systems, limiting this presentation to setting up the formalism to deal with ground state $(T=0)$ properties. The key new feature is that one must deal with energy functionals of both the local density $n(\mathbf{r})$ and the local complex macroscopic wavefunction $\Phi(\mathbf{r})$ associated with the Bose broken-symmetry (the local condensate density is $\left.n_{c}(\mathbf{r})=|\Phi(\mathbf{r})|^{2}\right)$. Implementing the Kohn-Sham scheme, we reduce the problem to a gas of weakly-interacting Bosons moving in self-consistent diagonal and off-diagonal one-body potentials. Our formalism should provide the basis for studies of the surface properties of liquid ${ }^{4} \mathrm{He}$ as well as the properties of Bose-condensed atomic gases trapped in external potentials.
\end{abstract}

Typeset using REVTEX 


\section{INTRODUCTION}

This article develops a density functional formalism for dealing with superfluid ${ }^{4} \mathrm{He}$, with the ultimate goal of understanding the role of Bose-condensation in the low density surface region. The use of neutron scattering to study the atomic structure and dynamics of superfluid ${ }^{4} \mathrm{He}$ has been an ongoing research effort at Chalk River since the early fifties. This world famous programme grew out of and was nurtured by the atmosphere which Bert Brockhouse helped to create. On a more personal note, I have always enjoyed being invited to give seminars at McMaster, partly for the stimulating discussions with research colleagues but also because I knew that Bert would be in the audience and after it was over, he would come over and give some encouraging comments. It is with great pleasure that I dedicate this article to Prof. Bert Brockhouse.

At a phenomenological level, the low temperature properties of bulk superfluid ${ }^{4} \mathrm{He}$ are well understood in terms of Landau's picture of a weakly-interacting quasiparticle gas of phonons and rotons. At a more microscopic (atomistic) level, current discussions of superfluid ${ }^{4} \mathrm{He}$ can be divided into two broad classes. One of these is the field-theoretic analysis, which is built on the fundamental role of Bose-broken symmetry. The superfluid phase coincides with the appearance of a macroscopic wavefunction given by the finite expectation value of the field operator $\Phi(\mathbf{r})=\langle\hat{\psi}(\mathbf{r})\rangle[\mathbb{1}$. This approach goes back to the pioneering work of Bogoliubov [2] and Penrose [3], but was first formulated in a systematic way by Beliaev [ [ 9 and Hugenholtz and Pines [5]. The current status of theories of superfluid ${ }^{4} \mathrm{He}$ based on the key role of the Bose order parameter $\Phi(\mathbf{r})$ is reviewed in a recent book [6].

A second kind of microscopic theory is built on the use of variational many-body wavefunctions for the ground state and the low-energy excited states of liquid ${ }^{4}$ He. Such manybody wavefunctions were first introduced by Bijl [7] and later by Feynman [B], and in their current form have become very sophisticated (the correlated basis function approach [9]). However, while such ground state many-particle wavefunctions (of the Jastrow-Feenberg type, for example) lead to very good estimates of the condensate fraction (about 10\% at 
$T=0$ ), the role of a Bose order parameter is not exhibited very explicitly (see chapter 9 of [6]).

The motivation of the present paper is to set up a formalism which can deal with the surface properties of superfluid ${ }^{4} \mathrm{He}$, taking Bose-condensation into account from the beginning. Formally this means we need a theory of spatially inhomogeneous Bose-condensed system. In the last decade, there has developed a considerable literature on superfluid ${ }^{4} \mathrm{He}$ with free surfaces (including droplets and films) based on generalizations of the above mentioned correlated basis function approach [10]. However, as with the case of bulk liquid ${ }^{4} \mathrm{He}$, such treatments of surfaces give little (if any) emphasis to the role of Bose-broken symmetry or Bose-Einstein condensation. On the other hand, the many-body wavefunctions which have been developed [11] to describe the free surface of liquid ${ }^{4} \mathrm{He}$ at $T=0$ appear to give realistic estimates of the density profile $n(\mathbf{r})$ in the surface region. From these, one finds accurate values of the binding energy of ${ }^{3} \mathrm{He}$ and spin-polarized $\mathrm{H}$ atoms bound to the surface of superfluid ${ }^{4} \mathrm{He}$ [12].

Such correlated basis function approaches involve a very heavy computational effort when dealing with inhomogeneous systems. An alternative, much simpler theory has developed for free surfaces of liquid ${ }^{4} \mathrm{He}$ based on a density functional theory [13]. This approach is loosely inspired by the very successful theory of inhomogeneous interacting electron systems and it is useful to recall some aspects of the latter theory. Almost 30 years ago, Hohenberg and Kohn (HK) gave a rigorous formulation [14] of the ground state properties of interacting Fermi systems, in terms of a functional of the local density $n(\mathbf{r})=\left\langle\hat{\psi}^{\dagger}(\mathbf{r}) \hat{\psi}(\mathbf{r})\right\rangle$. Kohn and Sham 15 (KS) used the two exact HK theorems to implement the HK formalism in terms of finding the single-particle energies and eigenstates of free Fermions moving in an appropriately defined self-consistent fields. This Hohenberg-Kohn-Sham (HKS) density functional formalism is now the accepted way of dealing with inhomogeneous Fermi systems, building on our extensive knowledge of the ground state properties of homogeneous interacting Fermi systems. The HKS approach has been generalized to deal with normal systems at finite temperatures [16] as well as BCS superconductors [17]. In the latter case, one works 
with functionals of the local density $n(\mathbf{r})$ and the local anomalous (or off-diagonal) density $\Delta(\mathbf{r}) \equiv\left\langle\hat{\psi}_{\uparrow}(\mathbf{r}) \hat{\psi}_{\downarrow}(\mathbf{r})\right\rangle$ describing spin-singlet Cooper pairs. Finally, a density functional formalism has been developed for dealing with time-dependent quantities (excited-states and linear response functions) of both normal [18] and superconducting Fermi systems [19].

As we have noted, recent density functional theories of superfluid ${ }^{4} \mathrm{He}$ with surfaces make analogies to the above HKS theory of inhomogeneous Fermi systems. However, there has apparently never been a careful study of how to use the HKS ideas to give a rigorous basis to a theory of inhomogeneous Bose-condensed liquids, analogous to what has been done for inhomogeneous BCS superconductors [17]. In particular, all current $T=0$ density functional theories of superfluid ${ }^{4} \mathrm{He}$ [13] simply assume that the energy functional only depends on the local density $n(\mathbf{r})$. There is never any reference to the possibly equally important role that the local condensate density $n_{c}(\mathbf{r})$ might play, or more generally, the local macroscopic wavefunction $\Phi(\mathbf{r})=\langle\hat{\psi}(\mathbf{r})\rangle=\sqrt{n_{c}(\mathbf{r})} e^{i S(\mathbf{r})}$. Needless to say, it is the finite value of the order parameter $\Phi(\mathbf{r})$ which characterizes the superfluid phase below $T_{\lambda}=2.17 \mathrm{~K}$. To avoid confusion, we note that the energy in density functional theories of liquid Helium (see third paper of ref. [13]) is often taken to be a functional of the variable $\Psi(\mathbf{r}) \equiv \sqrt{n(\mathbf{r})} e^{i S(\mathbf{r})}$, which involves the total local density. While this variable is sometimes referred to as a "macroscopic wavefunction", it is clearly unrelated to the Bose order parameter $\Phi(\mathbf{r})$ we have introduced above. The insufficiency of theories based on functionals of only $n(\mathbf{r})$ has been made especially obvious in recent work [20] which points out that the low density surface region of liquid ${ }^{4} \mathrm{He}$ corresponds to a dilute inhomogeneous Bose gas with 100\% Bose condensation (at $T=0$ ). In this surface region, the key function is $\Phi(\mathbf{r})$, with the local density being determined by it, namely $n(\mathbf{r})=n_{c}(\mathbf{r}) \equiv|\Phi(\mathbf{r})|^{2}$.

In the present paper, we formulate a density functional theory of the HKS kind for the ground state properties of an inhomogeneous interacting Bose-condensed fluid. The key new element in our analysis (following the analogous case of BCS superconductors [17]) is the realization that one must work with functionals of both $n(\mathbf{r})$ and the (complex) order parameter $\Phi(\mathbf{r})$. The key theorems of HK and the methods of proof are, of course, valid for 
Bose as well as Fermi statistics. For brevity, we shall only sketch these arguments when they involve the identical steps as in the density functional treatment of BCS superconductors. The present analysis of ground state properties can be extended to finite temperatures (free energies) following the analogous discussion for BCS superconductors [17]. This will be reported elsewhere.

What the present paper accomplishes is to give a formally exact scheme for dealing with inhomogeneous Bose-condensed fluids which should ultimately provide a platform for specific calculations. In applying the present formalism, one must introduce approximations for the correlation energy functionals. This is a separate question, with specific problems associated with the anomalous long-range correlations in Bose-condensed systems [21], and is not treated here.

In this paper, we mainly use the surface region of superfluid ${ }^{4} \mathrm{He}$ as an example of an inhomogeneous Bose-condensed system. Very recently, Bose-condensation has been finally achieved [22] in a dilute gas of ${ }^{87} \mathrm{Rb}$ atoms below $200 \mathrm{nK}$, using laser and evaporative cooling. This gas was trapped in a harmonic potential well and as a result, both $n(\mathbf{r})$ and $n_{c}(\mathbf{r})$ are highly inhomogeneous. Our present formalism gives a natural basis for generalizing the currently available Hartree-Fock-Bogoliubov approximations [23] for such inhomogeneous weakly interacting Bose-condensed gases.

\section{HOHENBERG-KOHN FORMALISM}

Our starting Hamiltonian is defined as (compare with [17])

$$
\begin{aligned}
\hat{H}_{v, \eta} \equiv & \int d \mathbf{r} \hat{\psi}^{\dagger}(\mathbf{r})\left[-\frac{\nabla^{2}}{2 m}-\mu\right] \hat{\psi}(\mathbf{r}) \\
& +\frac{1}{2} \int d \mathbf{r} \int d \mathbf{r}^{\prime} \hat{\psi}^{\dagger}(\mathbf{r}) \hat{\psi}^{\dagger}\left(\mathbf{r}^{\prime}\right) v_{2}\left(\mathbf{r}-\mathbf{r}^{\prime}\right) \hat{\psi}\left(\mathbf{r}^{\prime}\right) \hat{\psi}(\mathbf{r}) \\
& +\int d \mathbf{r} v(\mathbf{r}) \hat{\psi}^{\dagger}(\mathbf{r}) \hat{\psi}(\mathbf{r}) \\
& +\int d \mathbf{r}\left[\eta(\mathbf{r}) \hat{\psi}^{\dagger}(\mathbf{r})+\eta^{*}(\mathbf{r}) \hat{\psi}(\mathbf{r})\right] \\
\equiv & \hat{H}_{0}-\mu \hat{N}+\hat{V}_{2}+\hat{V}_{1}+\hat{V}_{S B} .
\end{aligned}
$$


Throughout the analysis, the two-particle interaction $v_{2}\left(\mathbf{r}-\mathbf{r}^{\prime}\right)$ is assumed to be fixed. Thus $\hat{H}_{v, \eta}$ in (1) depends only on: (a) the choice of the external diagonal single-particle potential $v(\mathbf{r})$, which couples to the density; and (b) the external off-diagonal (or symmetry-breaking) potential $\eta(\mathbf{r})$, which couples to the field operators $\hat{\psi}(\mathbf{r})$ and $\hat{\psi}^{\dagger}(\mathbf{r})$. The first step in the HK approach is to note that the ground state $|\Psi\rangle$ of $\hat{H}_{v, \eta}$ is a functional of these external fields $v(\mathbf{r})$ and $\eta(\mathbf{r})$, and hence so are the following groundstate expectation values:

$$
\begin{aligned}
n(\mathbf{r}) & \equiv\left\langle\Psi\left|\hat{\psi}^{\dagger}(\mathbf{r}) \hat{\psi}(\mathbf{r})\right| \Psi\right\rangle \\
\Phi(\mathbf{r}) & \equiv\langle\Psi|\hat{\psi}(\mathbf{r})| \Psi\rangle \\
\Phi^{*}(\mathbf{r}) & \equiv\left\langle\Psi\left|\hat{\psi}^{\dagger}(\mathbf{r})\right| \Psi\right\rangle .
\end{aligned}
$$

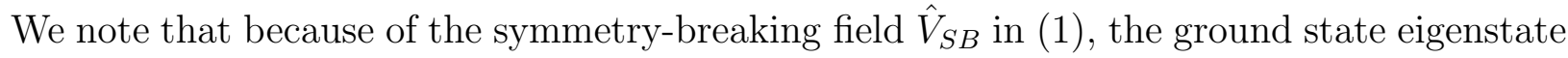
$|\Psi\rangle$ of $\hat{H}_{v, \eta}$ allows $\Phi(\mathbf{r})$ and $\Phi^{*}(\mathbf{r})$ to be finite. Introducing a symmetry-breaking (offdiagonal) perturbation as in (1) is the standard method [1, 1. of dealing with the appearance of Bose-condensation in an interacting system. It involves treating the condensate as a "reservoir" of atoms and thus leads to number non-conservation and to a groundstate where $\Phi(\mathbf{r})$ can be finite. The underlying physics of this broken symmetry is the same for both homogeneous and inhomogeneous systems and is discussed most clearly in Ref. [1]. We recall that the essential physics for dealing with BCS superconductors [17 also involves such symmetry-breaking number non-conserving states.

The second step of HK is to note that, using their famous reductio ad absurdum argument, that up to additive constants, one can prove

$$
v(\mathbf{r}) \text { and } \eta(\mathbf{r}) \text { are unique functionals of } n(\mathbf{r}) \text { and } \Phi(\mathbf{r}) .
$$

Since $v$ and $\eta$ fix $\hat{H}_{v, \eta}$ and thus also $|\Psi\rangle$, we can finally conclude that

$$
|\Psi\rangle \text { is a unique functional of } n(\mathbf{r}) \text { and } \Phi(\mathbf{r}) \text {. }
$$

In turn, it follows from (5) that the expectation value $\left\langle\Psi\left|\hat{H}_{0}-\mu \hat{N}+\hat{V}_{2}\right| \Psi\right\rangle$ is a universal functional of $n(\mathbf{r})$ and $\Phi(\mathbf{r})$. That is to say, there is no explicit dependence of $|\Psi\rangle$ on the 
specific forms assumed for $v(\mathbf{r})$ and $\eta(\mathbf{r})$, since these can be expressed as functionals of $n(\mathbf{r})$ and $\Phi(\mathbf{r})$, as stated in (14). Summarizing this train of argument, we conclude that

$$
F[n(\mathbf{r}), \Phi(\mathbf{r})] \equiv\left\langle\Psi\left|\hat{H}_{0}-\mu \hat{N}+\hat{V}_{2}\right| \Psi\right\rangle
$$

is a universal functional of $n$ and $\Phi$, valid for any number of particles and any external potentials $v(\mathbf{r})$ and $\eta(\mathbf{r})$. As in the HK formalism for Fermi systems, the universal functional $F[n, \Phi]$ will play a central role in our subsequent analysis. A key problem, of course, will be to find some appropriate approximation to this universal functional $F[n, \Phi]$ in superfluid ${ }^{4} \mathrm{He}$.

Following HK, it is useful to define, for given $v$ and $\eta$ potentials, the energy functional

$$
\begin{aligned}
E_{v, \eta}[n, \Phi] \equiv & F[n, \Phi]+\int d \mathbf{r} v(\mathbf{r}) n(\mathbf{r}) \\
& +\int d \mathbf{r}\left[\eta(\mathbf{r}) \Phi^{*}(\mathbf{r})+\eta^{*}(\mathbf{r}) \Phi(\mathbf{r})\right]
\end{aligned}
$$

where the densities $n(\mathbf{r}), \Phi(\mathbf{r})$ and $\Phi^{*}(\mathbf{r})$ are defined in (3). For simplicity of notation, we shall generally show functionals as depending only on $\Phi$, but in fact, they depend on both $\Phi$ and $\Phi^{*}$. Following HK, we have a variational principle, i.e., one can prove that $E_{v, \eta}[n, \Phi]$ is a minimum at the correct values of the densities $n(\mathbf{r})$ and $\Phi(\mathbf{r})$ produced by the external potentials $v(\mathbf{r})$ and $\eta(\mathbf{r})$, i.e.,

$$
\frac{\delta E_{v, \eta}[n, \Phi]}{\delta n(\mathbf{r})}=0, \frac{\delta E_{v, \eta}[n, \Phi]}{\delta \Phi(\mathbf{r})}=0 .
$$

\section{KOHN-SHAM PROCEDURE}

Following Kohn and Sham [15], there is a clever way of finding the correct values of $n(\mathbf{r})$ and $\Phi(\mathbf{r})$ (which, according to $(\mathbb{8})$, minimize $E_{v, \eta}[n, \Phi]$ ) by solving a simpler auxiliary problem for which the HK theorems in Section II are also valid. To understand the logic of the KS procedure in the context of our present problem, let us consider an auxiliary system Hamiltonian defined by 


$$
\begin{aligned}
\hat{H}_{v_{s}, \eta_{s}}^{s} \equiv & \int d \mathbf{r} \hat{\psi}^{\dagger}(\mathbf{r})\left[-\frac{\nabla^{2}}{2 m}-\mu\right] \hat{\psi}(\mathbf{r}) \\
& +\hat{V}_{s}\left[\hat{\psi}^{\dagger}, \hat{\psi}\right]+\int d \mathbf{r} v_{s}(\mathbf{r}) \hat{\psi}^{\dagger}(\mathbf{r}) \hat{\psi}(\mathbf{r}) \\
& +\int d \mathbf{r}\left[\eta_{s}(\mathbf{r}) \hat{\psi}^{\dagger}(\mathbf{r})+\eta_{s}^{*}(\mathbf{r}) \hat{\psi}(\mathbf{r})\right]
\end{aligned}
$$

where the interaction $\hat{V}_{s}$ is a part of $\hat{V}_{2}$ in (1) and (2), to be specified later. All the HK results of Section II apply to (9). In particular, if we denote the ground state of $\hat{H}_{v_{s}, \eta_{s}}^{s}$ as $\left|\Psi_{s}\right\rangle$, then the densities

$$
\begin{aligned}
n_{s}(\mathbf{r}) & \equiv\left\langle\Psi_{s}\left|\hat{\psi}^{\dagger}(\mathbf{r}) \hat{\psi}(\mathbf{r})\right| \Psi_{s}\right\rangle \\
\Phi_{s}(\mathbf{r}) & \equiv\left\langle\Psi_{s}|\hat{\psi}(\mathbf{r})| \Psi_{s}\right\rangle
\end{aligned}
$$

are unique functionals of the external fields $v_{s}(\mathbf{r})$ and $\eta_{s}(\mathbf{r})$. In turn, $v_{s}$ and $\eta_{s}$ and hence $\left|\Psi_{s}\right\rangle$ can be shown to be unique functionals of $n_{s}(\mathbf{r})$ and $\Phi_{s}(\mathbf{r})$. Thus we conclude that

$$
F_{s}\left[n_{s}(\mathbf{r}), \Phi_{s}(\mathbf{r})\right] \equiv\left\langle\Psi_{s}\left|\hat{H}_{o}-\mu \hat{N}+\hat{V}_{s}\right| \Psi_{s}\right\rangle
$$

is a universal functional of $n_{s}(\mathbf{r})$ and $\Phi_{s}(\mathbf{r})$. Finally, we can define an energy functional of this auxiliary system

$$
E_{v_{s}, \eta_{s}}^{s}[n, \Phi] \equiv F_{s}[n, \Phi]+\int d \mathbf{r} v_{s}(\mathbf{r}) n(\mathbf{r})+\int d \mathbf{r}\left[\eta_{s}(\mathbf{r}) \Phi^{*}(\mathbf{r})+\eta_{s}^{*}(\mathbf{r}) \Phi(\mathbf{r})\right]
$$

which will be minimized by the correct values, $n(\mathbf{r})=n_{s}(\mathbf{r})$ and $\Phi(\mathbf{r})=\Phi_{s}(\mathbf{r})$, for this system.

The whole point of introducing this auxiliary system defined by (9) is that:

(a) It will be easier to solve than the actual system described by (11).

(b) By a judicious choice of the external fields $v_{s}(\mathbf{r})$ and $\eta_{s}(\mathbf{r})$, the densities $n_{s}(\mathbf{r})$ and $\Phi_{s}(\mathbf{r})$ of this auxiliary problem can be made to be identical to those of the real system. Since $F[n(\mathbf{r}), \Phi(\mathbf{r})]$ in (6) is a universal functional of only $n(\mathbf{r})$ and $\Phi(\mathbf{r})$, this means that we can evaluate it using results for $n(\mathbf{r})$ and $\Phi(\mathbf{r})$ obtained from solving the auxiliary system. 
In applying the KS procedure to superconductors [17], one uses a non-interacting gas of Fermions moving in external one-body and pair potentials as the auxiliary model system. In our interacting Bose system, this would correspond to setting $V_{s}\left[\hat{\psi}^{\dagger}, \hat{\psi}\right]$ in (9) to zero. The problem with this choice is that for a non-interacting Bose gas moving in given external potentials $v_{s}$ and $\eta_{s}$, and at $T=0$, one has complete Bose-Einstein condensation (see discussion after (35) for more details). This implies that $n(\mathbf{r})$ and $n_{c}(\mathbf{r}) \equiv|\Phi(\mathbf{r})|^{2}$ are equal, even though we know that in any interacting Bose system, the local condensate density $n_{c}(\mathbf{r})$ is less than the local total density $n(\mathbf{r})$. This problem is not addressed in density functional theories 13 of superfluid ${ }^{4} \mathrm{He}$ based on functionals of only the density $n(\mathbf{r})$. We recall that such theories usually start with the kinetic energy of an inhomogeneous non-interacting Bose gas with a density profile $n(\mathbf{r})$ identical to the fully-interacting system. Such a kinetic energy functional implies that $n_{c}(\mathbf{r})=n(\mathbf{r})$, which would not appear to be a very good starting point for describing superfluid ${ }^{4} \mathrm{He}$.

In order to define our auxiliary Bose system in (9), we first introduce the usual decomposition of Bose quantum field operators [6]

$$
\begin{aligned}
\hat{\psi}(\mathbf{r}) & =\Phi(\mathbf{r})+\tilde{\psi}(\mathbf{r}) \\
\hat{\psi}^{\dagger}(\mathbf{r}) & =\Phi^{*}(\mathbf{r})+\tilde{\psi}^{\dagger}(\mathbf{r})
\end{aligned}
$$

where $\Phi(\mathbf{r})$ is defined in (3). The non-condensate field operators $\tilde{\psi}(\mathbf{r})$ and $\tilde{\psi}^{\dagger}(\mathbf{r})$ satisfy Bose commutation relations. Using (13), the two-particle interaction $\hat{V}_{2}$ in (11) can be rewritten as

$$
\begin{aligned}
\hat{V}_{2}= & \frac{1}{2} \int d \mathbf{r} \int d \mathbf{r}^{\prime} v_{2}\left(\mathbf{r}-\mathbf{r}^{\prime}\right)|\Phi(\mathbf{r})|^{2}\left|\Phi\left(\mathbf{r}^{\prime}\right)\right|^{2} \\
& +\int d \mathbf{r} \int d \mathbf{r}^{\prime} v_{2}\left(\mathbf{r}-\mathbf{r}^{\prime}\right)|\Phi(\mathbf{r})|^{2} \Phi\left(\mathbf{r}^{\prime}\right) \tilde{\psi}^{\dagger}\left(\mathbf{r}^{\prime}\right) \\
& +\int d \mathbf{r} \int d \mathbf{r}^{\prime} v_{2}\left(\mathbf{r}-\mathbf{r}^{\prime}\right)|\Phi(\mathbf{r})|^{2} \Phi^{*}\left(\mathbf{r}^{\prime}\right) \tilde{\psi}\left(\mathbf{r}^{\prime}\right) \\
& +\int d \mathbf{r} \int d \mathbf{r}^{\prime} v_{2}\left(\mathbf{r}-\mathbf{r}^{\prime}\right)\left[\tilde{\psi}^{\dagger}(\mathbf{r}) \tilde{\psi}(\mathbf{r})\left|\Phi\left(\mathbf{r}^{\prime}\right)\right|^{2}+\tilde{\psi}^{\dagger}(\mathbf{r}) \tilde{\psi}\left(\mathbf{r}^{\prime}\right) \Phi^{*}\left(\mathbf{r}^{\prime}\right) \Phi(\mathbf{r})\right. \\
& \left.+\frac{1}{2} \tilde{\psi}^{\dagger}(\mathbf{r}) \tilde{\psi}^{\dagger}\left(\mathbf{r}^{\prime}\right) \Phi\left(\mathbf{r}^{\prime}\right) \Phi(\mathbf{r})+\frac{1}{2} \tilde{\psi}(\mathbf{r}) \tilde{\psi}\left(\mathbf{r}^{\prime}\right) \Phi^{*}\left(\mathbf{r}^{\prime}\right) \Phi^{*}(\mathbf{r})\right] \\
& +\int d \mathbf{r} \int d \mathbf{r}^{\prime} v_{2}\left(\mathbf{r}-\mathbf{r}^{\prime}\right)\left[\tilde{\psi}^{\dagger}\left(\mathbf{r}^{\prime}\right) \tilde{\psi}\left(\mathbf{r}^{\prime}\right) \tilde{\psi}(\mathbf{r}) \Phi^{*}(\mathbf{r})+\tilde{\psi}^{\dagger}\left(\mathbf{r}^{\prime}\right) \tilde{\psi}^{\dagger}(\mathbf{r}) \tilde{\psi}\left(\mathbf{r}^{\prime}\right) \Phi(\mathbf{r})\right]
\end{aligned}
$$




$$
+\frac{1}{2} \int d \mathbf{r} \int d \mathbf{r}^{\prime} v_{2}\left(\mathbf{r}-\mathbf{r}^{\prime}\right) \tilde{\psi}^{\dagger}(\mathbf{r}) \tilde{\psi}^{\dagger}\left(\mathbf{r}^{\prime}\right) \tilde{\psi}\left(\mathbf{r}^{\prime}\right) \tilde{\psi}(\mathbf{r})
$$

If all the atoms were Bose-condensed, only the first term in (14) would be important. If the system is not Bose-condensed, then only the last term in (14) is present. In the well-known Bogoliubov approximation [24,25] for a dilute, weakly interacting gas, in which almost all the atoms are Bose-condensed, one only keeps terms up to quadratic in the non-condensate field operators $\tilde{\psi}$ and $\tilde{\psi}^{\dagger}$ (since it is assumed that, in an average sense, $\tilde{\psi} \ll \Phi$ ). That is to say, the last two terms in (14) are higher order and hence omitted. A feature of this Bogoliubov approximation is that the resulting Hamiltonian can be diagonalized exactly (see below).

We now define what we shall call (for want of a better term) the exchange-correlation energy functional $F_{x c}[n, \Phi]$ by writing (6) in the form

$$
F[n, \Phi]=F_{s}[n, \Phi]+\frac{1}{2} \int d \mathbf{r} \int d \mathbf{r}^{\prime} v_{2}\left(\mathbf{r}-\mathbf{r}^{\prime}\right) n(\mathbf{r}) n\left(\mathbf{r}^{\prime}\right)+F_{x c}[n, \Phi],
$$

where $F_{s}[n, \Phi]$ is the energy functional of the auxiliary system defined by (11) with the interaction

$$
\begin{aligned}
\hat{V}_{s}= & \int d \mathbf{r} \int d \mathbf{r}^{\prime} v_{2}\left(\mathbf{r}-\mathbf{r}^{\prime}\right)|\Phi(\mathbf{r})|^{2}\left[\Phi\left(\mathbf{r}^{\prime}\right) \tilde{\psi}^{\dagger}\left(\mathbf{r}^{\prime}\right)+\Phi^{*}\left(\mathbf{r}^{\prime}\right) \tilde{\psi}\left(\mathbf{r}^{\prime}\right)\right] \\
& +\frac{1}{2} \int d \mathbf{r} \int d \mathbf{r}^{\prime} v_{2}\left(\mathbf{r}-\mathbf{r}^{\prime}\right)\left[2 \Phi^{*}\left(\mathbf{r}^{\prime}\right) \Phi(\mathbf{r}) \tilde{\psi}^{\dagger}(\mathbf{r}) \tilde{\psi}\left(\mathbf{r}^{\prime}\right)\right. \\
& \left.+\Phi\left(\mathbf{r}^{\prime}\right) \Phi(\mathbf{r}) \tilde{\psi}^{\dagger}(\mathbf{r}) \tilde{\psi}^{\dagger}\left(\mathbf{r}^{\prime}\right)+\Phi^{*}\left(\mathbf{r}^{\prime}\right) \Phi^{*}(\mathbf{r}) \tilde{\psi}(\mathbf{r}) \tilde{\psi}\left(\mathbf{r}^{\prime}\right)\right]
\end{aligned}
$$

and subject to potentials $v_{s}(\mathbf{r})$ and $\eta_{s}(\mathbf{r})$ [see ([12)] chosen such that the density $n_{s}(\mathbf{r})$ and order parameter $\Phi_{s}(\mathbf{r})$ are identical to those of the full system. As usual [14,15], it is useful to separate out the total Hartree energy contribution as we have done in (15). Writing this contribution out more explicitly for a Bose-condensed system, we have

$$
\left\langle\hat{V}_{H}\right\rangle=\frac{1}{2} \int d \mathbf{r} \int d \mathbf{r}^{\prime} v_{2}\left(\mathbf{r}-\mathbf{r}^{\prime}\right)\left[|\Phi(\mathbf{r})|^{2}\left|\Phi\left(\mathbf{r}^{\prime}\right)\right|^{2}+2|\Phi(\mathbf{r})|^{2} \tilde{n}\left(\mathbf{r}^{\prime}\right)+\tilde{n}(\mathbf{r}) \tilde{n}\left(\mathbf{r}^{\prime}\right)\right]
$$

where the non-condensate local density is defined by

$$
\tilde{n}(\mathbf{r}) \equiv\left\langle\tilde{\psi}^{\dagger}(\mathbf{r}) \tilde{\psi}(\mathbf{r})\right\rangle=n(\mathbf{r})-|\Phi(\mathbf{r})|^{2}
$$


The last two terms in (17) come from the $\tilde{\psi}^{\dagger} \tilde{\psi}$ and $\left(\tilde{\psi}^{\dagger} \tilde{\psi}\right)^{2}$ terms in (14).

Calculating the variational derivatives in (8) using $F[n, \Phi]$ in (15), one finds

$$
\begin{aligned}
\frac{\delta F_{s}[n, \Phi]}{\delta n(\mathbf{r})}+v_{H}(\mathbf{r})+\frac{\delta F_{x c}[n, \Phi]}{\delta n(\mathbf{r})}+v(\mathbf{r}) & =0 \\
\frac{\delta F_{s}[n, \Phi]}{\delta \Phi(\mathbf{r})}+\frac{\delta F_{x c}[n, \Phi]}{\delta \Phi(\mathbf{r})}+\eta(\mathbf{r}) & =0
\end{aligned}
$$

where the Hartree field is defined as

$$
v_{H}(\mathbf{r}) \equiv \int d \mathbf{r}^{\prime} v_{2}\left(\mathbf{r}-\mathbf{r}^{\prime}\right) n\left(\mathbf{r}^{\prime}\right)
$$

Similarly, using (8) for the auxiliary system defined above, one finds

$$
\begin{aligned}
& \frac{\delta F_{s}[n, \Phi]}{\delta n(\mathbf{r})}+v_{s}(\mathbf{r})=0 \\
& \frac{\delta F_{s}[n, \Phi]}{\delta \Phi(\mathbf{r})}+\eta_{s}(\mathbf{r})=0
\end{aligned}
$$

Combining the results in (19) and (21), we conclude that the density and order parameters of the auxiliary system will be identical with the actual system if

$$
\begin{aligned}
& v_{s}(\mathbf{r})=v(\mathbf{r})+v_{H}(\mathbf{r})+\frac{\delta F_{x c}[n, \Phi]}{\delta n(\mathbf{r})}=v_{s}[n, \Phi] \\
& \eta_{s}(\mathbf{r})=\eta(\mathbf{r})+\frac{\delta F_{x c}[n, \Phi]}{\delta \Phi(\mathbf{r})}=\eta_{s}[n, \Phi] .
\end{aligned}
$$

This gives $v_{s}$ and $\eta_{s}$ as explicit functionals of $n(\mathbf{r})$ and $\Phi(\mathbf{r})$, once we have decided on a specific form for the functional $F_{x c}[n, \Phi]$.

\section{BOGOLIUBOV GAS AS AUXILIARY SYSTEM}

We now turn to the auxiliary system defined by (9), with $\hat{V}_{s}$ given by (16). Using (13), one finds that

$$
\begin{aligned}
\hat{H}_{\eta_{s}, v_{s}}^{s}= & \int d \mathbf{r} \Phi^{*}(\mathbf{r})\left[\hat{L} \Phi(\mathbf{r})+\eta_{s}(\mathbf{r})\right]+\int d \mathbf{r} \eta_{s}^{*}(\mathbf{r}) \Phi(\mathbf{r}) \\
& +\int d \mathbf{r} \tilde{\psi}^{\dagger}(\mathbf{r})\left[\hat{L} \Phi(\mathbf{r})+\eta_{s}(\mathbf{r})\right] \\
& +\int d \mathbf{r}\left[\Phi^{*}(\mathbf{r}) \hat{L}+\eta_{s}^{*}(\mathbf{r})\right] \tilde{\psi}(\mathbf{r})
\end{aligned}
$$




$$
\begin{aligned}
& +\int d \mathbf{r} \tilde{\psi}^{\dagger}(\mathbf{r}) \hat{L} \tilde{\psi}(\mathbf{r}) \\
& +\frac{1}{2} \int d \mathbf{r} \int d \mathbf{r}^{\prime} v_{2}\left(\mathbf{r}-\mathbf{r}^{\prime}\right)\left[2 \Phi^{*}\left(\mathbf{r}^{\prime}\right) \Phi(\mathbf{r}) \tilde{\psi}^{\dagger}(\mathbf{r}) \tilde{\psi}\left(\mathbf{r}^{\prime}\right)\right. \\
& \left.+\Phi\left(\mathbf{r}^{\prime}\right) \Phi(\mathbf{r}) \tilde{\psi}^{\dagger}(\mathbf{r}) \tilde{\psi}^{\dagger}\left(\mathbf{r}^{\prime}\right)+\Phi^{*}\left(\mathbf{r}^{\prime}\right) \Phi^{*}(\mathbf{r}) \tilde{\psi}(\mathbf{r}) \tilde{\psi}\left(\mathbf{r}^{\prime}\right)\right]
\end{aligned}
$$

where the operator $\hat{L}$ is defined by

$$
\hat{L} \equiv\left(-\frac{\nabla^{2}}{2 m}+v_{s}(\mathbf{r})-\mu\right)
$$

This Hamiltonian is similar in structure to the one one obtains in an inhomogeneous weakly interacting Bose gas at $T=0$, which has been extensively studied in the literature [24,25]. We solve it using similar techniques. In order to diagonalize (23), we first eliminate the terms linear in $\tilde{\psi}$ and $\tilde{\psi}^{\dagger}$ by requiring that $\Phi(\mathbf{r})$ satisfy the equation

$$
\hat{L} \Phi(\mathbf{r})+\eta_{s}(\mathbf{r})=0
$$

Eq. (25) is a sort of generalized Gross-Pitaevskii equation for $\Phi$, but now in the context of density functional theory rather than for a dilute Bose gas. This connection is easily seen by setting $F_{x c}[n, \Phi]$ in (15) to zero, in which case (22) simplifies to

$$
\begin{aligned}
& v_{s}(\mathbf{r})=v(\mathbf{r})+v_{H}(\mathbf{r}) \\
& \eta_{s}(\mathbf{r})=\eta(\mathbf{r}),
\end{aligned}
$$

and (25) reduces to

$$
\left[-\frac{\nabla^{2}}{2 m}+v(\mathbf{r})-\mu+\int d \mathbf{r}^{\prime} v_{2}\left(\mathbf{r}-\mathbf{r}^{\prime}\right) n\left(\mathbf{r}^{\prime}\right)\right] \Phi(\mathbf{r})+\eta(\mathbf{r})=0 .
$$

Setting the external fields $v$ and $\eta$ to zero, we recover the well-known Gross-Pitaevskii equation 26.24 for a dilute inhomogeneous gas at $T=0$. In that case, since all the atoms are Bose-condensed, the density $n(\mathbf{r})$ in (27) can be approximated by $n_{c}(\mathbf{r})=|\Phi(\mathbf{r})|^{2}$, and then (27) is a closed non-linear Schrodinger equation (NLSE) for $\Phi(\mathbf{r})$.

Assuming that $\Phi(\mathbf{r})$ satisfies (25), our auxiliary system Hamiltonian (23) reduces to 


$$
\begin{aligned}
\hat{H}_{\eta_{s}, v_{s}}^{s}= & \int d \mathbf{r} \eta_{s}^{*}(\mathbf{r}) \Phi(\mathbf{r}) \\
& +\int d \mathbf{r} \tilde{\psi}^{\dagger}(\mathbf{r})\left[-\frac{\nabla^{2}}{2 m}+v_{s}(\mathbf{r})-\mu\right] \tilde{\psi}(\mathbf{r}) \\
& +\int d \mathbf{r} \int d \mathbf{r}^{\prime} v_{2}\left(\mathbf{r}-\mathbf{r}^{\prime}\right) \Phi^{*}\left(\mathbf{r}^{\prime}\right) \Phi(\mathbf{r}) \tilde{\psi}^{\dagger}(\mathbf{r}) \tilde{\psi}\left(\mathbf{r}^{\prime}\right) \\
& +\frac{1}{2} \int d \mathbf{r} \int d \mathbf{r}^{\prime} v_{2}\left(\mathbf{r}-\mathbf{r}^{\prime}\right)\left[\Phi\left(\mathbf{r}^{\prime}\right) \Phi(\mathbf{r}) \tilde{\psi}^{\dagger}(\mathbf{r}) \tilde{\psi}^{\dagger}\left(\mathbf{r}^{\prime}\right)+\Phi^{*}\left(\mathbf{r}^{\prime}\right) \Phi^{*}(\mathbf{r}) \tilde{\psi}(\mathbf{r}) \tilde{\psi}\left(\mathbf{r}^{\prime}\right)\right]
\end{aligned}
$$

The Hartree contribution is contained in $v_{s}(\mathbf{r})$ [see (22)]. The third line in (28) gives the exchange term, while the fourth line involves the anomalous contributions involving $\tilde{\psi}^{\dagger} \tilde{\psi}^{\dagger}$ and $\tilde{\psi} \tilde{\psi}$ characteristic of a Bose-condensed system. The quadratic expression given by (28) can be diagonalized by the usual Bogoliubov transformation [25]

$$
\begin{aligned}
\tilde{\psi}(\mathbf{r}) & =\sum_{j}\left[u_{j}(\mathbf{r}) \alpha_{j}-v_{j}^{*}(\mathbf{r}) \alpha_{j}^{\dagger}\right] \\
\tilde{\psi}^{\dagger}(\mathbf{r}) & =\sum_{j}\left[u_{j}^{*}(\mathbf{r}) \alpha_{j}^{\dagger}-v_{j}(\mathbf{r}) \alpha_{j}\right]
\end{aligned}
$$

where the new "quasiparticle" operators $\alpha_{j}$ and $\alpha_{j}^{\dagger}$ satisfy Bose commutation relations. One finds the amplitudes $u_{j}(\mathbf{r})$ and $v_{j}(\mathbf{r})$ are given by the generalized Bogoliubov coupled equations:

$$
\begin{aligned}
{\left[-\frac{\nabla^{2}}{2 m}+v_{s}(\mathbf{r})-\mu\right] u_{j}(\mathbf{r}) } & +\int d \mathbf{r}^{\prime} v_{2}\left(\mathbf{r}-\mathbf{r}^{\prime}\right) \Phi(\mathbf{r}) \Phi^{*}\left(\mathbf{r}^{\prime}\right) u_{j}\left(\mathbf{r}^{\prime}\right) \\
& -\int d \mathbf{r}^{\prime} v_{2}\left(\mathbf{r}-\mathbf{r}^{\prime}\right) \Phi(\mathbf{r}) \Phi\left(\mathbf{r}^{\prime}\right) v_{j}\left(\mathbf{r}^{\prime}\right)=E_{j} u_{j}(\mathbf{r}) \\
{\left[-\frac{\nabla^{2}}{2 m}+v_{s}(\mathbf{r})-\mu\right] v_{j}(\mathbf{r})+} & \int d \mathbf{r}^{\prime} v_{2}\left(\mathbf{r}-\mathbf{r}^{\prime}\right) \Phi^{*}(\mathbf{r}) \Phi\left(\mathbf{r}^{\prime}\right) v_{j}\left(\mathbf{r}^{\prime}\right) \\
- & \int d \mathbf{r}^{\prime} v_{2}\left(\mathbf{r}-\mathbf{r}^{\prime}\right) \Phi^{*}(\mathbf{r}) \Phi^{*}\left(\mathbf{r}^{\prime}\right) u_{j}\left(\mathbf{r}^{\prime}\right)=-E_{j} v_{j}(\mathbf{r}) .
\end{aligned}
$$

One can prove that the eigenvalues $E_{j}$ are real and that one must choose solutions such that $E_{j} \geq 0$. For details of how (30) is derived, we refer to a similar calculation by Fetter 25 for the case of a contact interaction $v_{2}(\mathbf{r})=v_{0} \delta(\mathbf{r})$. However, we note that long-range tail of the He-He interatomic potential is very important when dealing with the surface region of liquid Helium [20].

With $u_{j}$ and $v_{j}$ given by the solutions of (30), the Hamiltonian in (28) can be shown to reduce to 


$$
\hat{H}_{\eta_{s}, v_{s}}^{s}=\int d \mathbf{r} \eta_{s}^{*}(\mathbf{r}) \Phi(\mathbf{r})-\sum_{j} E_{j} \int d \mathbf{r}\left|v_{j}(\mathbf{r})\right|^{2}+\sum_{j} E_{j} \hat{\alpha}_{j}^{\dagger} \hat{\alpha}_{j}
$$

which describes a non-interacting gas of quasiparticles of energy $E_{j}$. The ground state $\left|\Psi_{s}\right\rangle$ of this Hamiltonian is defined by $\hat{\alpha}_{j}\left|\Psi_{s}\right\rangle=0$. Thus the ground state expectation value of (31) is given by

$$
E_{\eta_{s}, v_{s}}^{s}=\int d \mathbf{r} \eta_{s}^{*}(\mathbf{r}) \Phi(\mathbf{r})-\sum_{j} E_{j} \int d \mathbf{r}\left|v_{j}(\mathbf{r})\right|^{2}
$$

Using (29), the non-condensate local density in (18) is given by $(T=0)$

$$
\begin{aligned}
\tilde{n}(\mathbf{r}) & \equiv\left\langle\Psi_{s}\left|\tilde{\psi}^{\dagger}(\mathbf{r}) \tilde{\psi}(\mathbf{r})\right| \Psi_{s}\right\rangle \\
& =\sum_{j}\left|v_{j}(\mathbf{r})\right|^{2} .
\end{aligned}
$$

Inserting (31) into (12) and using (25) and (18), one finds after a little algebra that

$$
F_{s}[n, \Phi]=\int d \mathbf{r} \Phi^{*}(\mathbf{r})\left[-\frac{\nabla^{2}}{2 m}-\mu\right] \Phi(\mathbf{r})-\sum_{j} \int d \mathbf{r}\left|v_{j}(\mathbf{r})\right|^{2}\left[E_{j}+v_{s}(\mathbf{r})\right] .
$$

We recall that the Hartree contribution was separated out in (15) and consequently it is not contained in $\hat{H}_{\eta_{s}, v_{s}}^{s}$ defined in (23). Thus it is not included in the energy eigenvalues $E_{j}$ given by (30), but rather appears as a separate contribution from the diagonal potential $v_{s}(\mathbf{r})$ in $(22)$.

The key feature of the above results is that one can have a depletion of the condensate, as shown by the finite value of $\tilde{n}(\mathbf{r})$ in (33). Thus the auxiliary system defined by (23) and (22) can be used to find both $\Phi(\mathbf{r})$ and $n(\mathbf{r})$ [using (25) and (30)] even when $n_{c}(\mathbf{r}) \equiv|\Phi(\mathbf{r})|^{2}$ and $n(\mathbf{r})$ are quite different (as in superfluid ${ }^{4} \mathrm{He}$ ).

If we had chosen a non-interacting Bose gas as our KS reference system $\left[i . e\right.$., set $\hat{V}_{s}=0$ in (9)], the last term in (23) would be absent. The linear terms in $\tilde{\psi}$ and $\tilde{\psi}^{\dagger}$ can be eliminated as before by requiring that $\Phi(\mathbf{r})$ satisfy (25). Then $\hat{H}_{\eta_{s}, v_{s}}^{s}$ is easily diagonalized, with $u_{j}(\mathbf{r})$ given by the solution

$$
\left(-\frac{\nabla^{2}}{2 m}+v_{s}(\mathbf{r})-\mu\right) u_{j}(\mathbf{r})=E_{j} u_{j}(\mathbf{r})
$$


and $v_{j}(\mathbf{r})=0$. It immediately follows from (33) that such a KS reference system leads to no depletion, with $\tilde{n}(\mathbf{r})=0$. This means that using a free Bose gas as a reference system inevitably leads to $n_{c}(\mathbf{r}) \equiv|\Phi(\mathbf{r})|^{2}$ and $n(\mathbf{r})$ being identical, no matter what we choose for the functional $F_{x c}[n, \Phi]$. This apparent "insufficiency" of the non-interacting Bose gas is somewhat surprising and deserves further study. It seems to be associated with the well-known fact that a weakly interacting Bose-condensed gas has qualitatively different properties than an ideal Bose gas.

It is convenient at this point to summarize the various steps in the KS procedure:

(1) One chooses some approximation for the "exchange-correlation" functional $F_{x c}[n, \Phi]$ defined in (15), giving it as an explicit functional of the local quantities $n(\mathbf{r})$ and $\Phi(\mathbf{r})$. This is the big step containing the "physics", and has not been addressed in the present paper.

(2) The potentials $v_{s}(\mathbf{r})$ and $\eta_{s}(\mathbf{r})$ are then computed using (22) and given as functionals of $n(\mathbf{r})$ and $\Phi(\mathbf{r})$.

(3) Evaluating $v_{s}$ and $\eta_{s}$ using an assumed (or trial) value of $n(\mathbf{r})$, the GP-type equation (25) is solved for $\Phi(\mathbf{r})$, i.e.,

$$
\left[-\frac{\nabla^{2}}{2 m}+v_{s}[n, \Phi]-\mu\right] \Phi(\mathbf{r})+\eta_{s}[n, \Phi]=0 .
$$

(4) Evaluating $v_{s}[n, \Phi]$ using the trial $n(\mathbf{r})$ and the solution for $\Phi(\mathbf{r})$ given in step (3), the generalized Bogoliubov equations in (30) can be solved to determine $E_{j}, u_{j}(\mathbf{r})$ and $v_{j}(\mathbf{r})$.

(5) With these results, one can calculate the non-condensate local density $\tilde{n}(\mathbf{r})$ in (33) and hence finally obtain $n(\mathbf{r})$ from (18), namely

$$
n(\mathbf{r})=|\Phi(\mathbf{r})|^{2}+\sum_{j}\left|v_{j}(\mathbf{r})\right|^{2} .
$$

Using this new expression for $n(\mathbf{r})$, one can go back to step (3) and repeat the procedure, until self-consistent values of $\Phi(\mathbf{r})$ and $n(\mathbf{r})$ are obtained. 
(6) These values of $n(\mathbf{r})$ and $\Phi(\mathbf{r})$ are then inserted into the energy functional of the actual Bose system of interest, as given by (7) and (15).

\section{CONCLUSIONS}

As in the case of interacting Fermi systems [15],17], it is important to emphasize that while the auxiliary Bose system described in Section IV corresponds to a dilute, weakly interacting Bose gas, we are only using it to find the local density $n(\mathbf{r})$ and local order parameter $\Phi(\mathbf{r})$ of a Bose-condensed liquid. In particular, the quasiparticle excitations which are described by the generalized Bogoliubov equations of motion in (30) have, in general, no "direct" physical significance in the true system.

The above procedure gives a well-defined scheme to find (at $T=0$ ) the energy, local density and local condensate density in superfluid ${ }^{4} \mathrm{He}$ with a free surface, once one has chosen some explicit approximation for the exchange-correlation functional $F_{x c}[n, \Phi]$ in (15). As in the case of normal and superconducting metals, a good approximation for this functional is the key to obtaining reasonable results using the density functional formalism. We hope to discuss this problem elsewhere. In the case of superfluid ${ }^{4} \mathrm{He}$, there has been considerable work 13 on constructing functionals which only depend on the total local density $n(\mathbf{r})$, in which one tries to build in known experimental information (compressibility, ground state energy, surface tension, etc.). In developing the equivalent approximations for use in our new formalism, the first thing we need to understand better is the ground-state energy of bulk liquid ${ }^{4} \mathrm{He}$ as a function of the density $n$ and the condensate density $n_{0}$. More Monte Carlo calculations would be very useful.

One immediate implication of the present theory is contained in the first term of (34), which can be rewritten in the form (for clarity, we insert $\hbar$ )

$$
\int d \mathbf{r} \Phi^{*}(\mathbf{r})\left[-\frac{\hbar^{2} \nabla^{2}}{2 m}\right] \Phi(\mathbf{r})=\int d \mathbf{r} \frac{\hbar^{2}}{2 m}\left|\nabla \sqrt{n_{c}(\mathbf{r})}\right|^{2}+\frac{1}{2} \int d \mathbf{r} m n_{c}(\mathbf{r}) \mathbf{v}_{s}^{2}(\mathbf{r})
$$

where we have used $\Phi(\mathbf{r})=\sqrt{n_{c}(\mathbf{r})} e^{i S(\mathbf{r})}$ and $m \mathbf{v}_{s}(\mathbf{r}) \equiv \hbar \nabla S(\mathbf{r})$ is the local superfluid 
velocity (in the ground state, we can set $\mathbf{v}_{s}(\mathbf{r})=0$ ). In contrast with the first term in (38), currently available density functional theories [13] always start with a term of the form

$$
\int d \mathbf{r} \frac{\hbar^{2}}{2 m}|\nabla \sqrt{n(\mathbf{r})}|^{2}
$$

involving the total local density $n(\mathbf{r})$. This corresponds to the kinetic energy functional of an inhomogeneous non-interacting Bose gas constrained to have the correct local density of the Bose liquid under consideration. We believe that the first term in (38) has a more natural as well as more sound theoretical basis when dealing with Bose-condensed fluids, and should be used in developing improved density functional theories of superfluid ${ }^{4} \mathrm{He}$ [20].

At the present time, the only detailed discussion of the properties of a spatially inhomogeneous Bose-condensed system has been for a weakly interacting atomic gas trapped in an external potential well [23]. In such systems [22], the condensate density $n_{c}(\mathbf{r})$ is strongly peaked at the center of the trap (due to the macroscopic occupation of the lowest quantum state) and has a very different spatial dependence from the non-condensate density $\tilde{n}(\mathbf{r})$. Our density functional formalism (extended to finite temperatures) should be useful in calculating the properties of such trapped Bose-condensed gases.

\section{ACKNOWLEDGEMENTS}

I would like to acknowledge many stimulating discussions with Sandro Stringari, which led to this work. I also thank Hardy Gross and the referee for useful comments. This work was done while on sabbatical at the University of Trento, which provided financial support and a congenial atmosphere. I also thank NSERC for a research grant. 


\section{REFERENCES}

[1] P.C. Hohenberg and P.C. Martin, Ann. Physics (N.Y.), 34, 291 (1965).

[2] N.N. Bogoliubov, J. Phys. U.S.S.R. 11, 23 (1947).

[3] O. Penrose, Phil. Mag. 42, 1373 (1951).

[4] S.T. Beliaev, Sov. Phys.-JETP 7, 289 (1958).

[5] N. Hugenholtz and D. Pines, Phys. Rev. 116, 489 (1959).

[6] A. Griffin, Excitations in a Bose-Condensed Liquid (Cambridge, N.Y., 1993).

[7] A. Bijl, Physica 7, 869 (1940).

[8] R.P. Feynman, Phys. Rev. 94, 262 (1954).

[9] E. Feenberg, Theory of Quantum Liquids (Academic, N.Y., 1969); C.E. Campbell, in Progress in Liquid Physics, ed. by C.A. Croxton (Wiley, London, 1977), ch. 6.

[10] For references, see E. Krotscheck, G.-X. Qian and W. Kohn, Phys. Rev. B31, 4245 (1985); K.A. Gernoth, J.W. Clark, G. Senger and M.L. Ristig, Phys. Rev. B49, 15836 (1994).

[11] See, for example, C.C. Chang and M. Cohen, Phys. Rev. A8, 3131 (1973).

[12] I.B. Mantz and D.O. Edwards, Phys. Rev. B20, 4518 (1979).

[13] C. Ebner and W.F. Saam, Phys. Rev. B12, 923 (1975); S. Stringari and J. Treiner, Phys. Rev. B36, 8369 (1987); for more recent references, see F. Dalfovo, A. Lastri, L. Pricaupenko, S. Stringari and J. Treiner, Phys. Rev. B52, 1193 (1995) .

[14] P. Hohenberg and W. Kohn, Phys. Rev. B136, 864 (1964).

[15] W. Kohn and L.J. Sham, Phys. Rev. A140, 1133 (1965).

[16] D. Mermin, Phys. Rev. 137, A1441 (1965). 
[17] L.N. Oliveira, E.K.U. Gross and W. Kohn, Phys. Rev. Letts. 60, 2430 (1988).

[18] E. Runge and E.K.U. Gross, Phys. Rev. Letts. 52, 997 (1984).

[19] O.J. Wacker, R. Kümmel and E.K.U. Gross, Phys. Rev. Letts. 73, 2915 (1994).

[20] A. Griffin and S. Stringari, Phys. Rev. Letts., in press.

[21] Y.A. Nepomnyashchy and L.P. Pitaevskii, Physica B194-196, 543 (1994).

[22] M.H. Anderson, J.R. Ensher, M.R. Matthews, C.E. Wieman and E.A. Cornell, Science 269, 198 (1995).

[23] V.V. Goldman, I.F. Silvera and A.J. Leggett, Phys. Rev. B24, 2870 (1981); D.A. Huse and E.D. Siggia, Journ. Low Temp. Phys. 46, 137 (1982).

[24] A.L. Fetter and J.D. Walecka, Quantum Theory of Many-Particle Systems (McGrawHill, N.Y., 1971), p. 492ff.

[25] A.L. Fetter, Am. Phys. (N.Y.) 70, 67 (1972).

[26] L.P. Pitaevskii, Sov. Phys. JETP 13, 451 (1961); E.P. Gross, Nuovo Cimento 20, 454 (1961). 